\title{
BplMYB46 from Betula platyphylla Can Form Homodimers and Heterodimers and Is Involved in Salt and Osmotic Stresses
}

\author{
Yan-Min Wang ${ }^{1,2}$, Chao Wang ${ }^{1}$, Hui-Yan Guo ${ }^{3, *}$ and Yu-Cheng Wang ${ }^{1,3, *}$ \\ 1 State Key Laboratory of Tree Genetics and Breeding, Northeast Forestry University, Harbin 150040, China; \\ wangyanmin1919@163.com (Y.-M.W.); wzyrgm@163.com (C.W.) \\ 2 Key Laboratory of Fast-Growing Tree Cultivating of Heilongiang Province, Forestry Science Research \\ Institute of Heilongjiang Province, Harbin 150040, China \\ 3 College of Forestry, Shenyang Agricultural University, Shenyang 110866, China \\ * Correspondence: wsghy1307@126.com (H.-Y.G.); wangyucheng@ms.xjb.ac.cn (Y.-C.W.)
}

Received: 11 January 2019; Accepted: 4 March 2019; Published: 7 March 2019

check for updates

\begin{abstract}
MYB proteins play important roles in the regulation of plant growth, development, and stress responses. Overexpression of BplMYB46 from Betula platyphylla improved plant salt and osmotic tolerances. In the present study, the interaction of eight avian myeloblastosis viral oncogene homolog (MYB) transcription factors with BplMYB46 was investigated using the yeast two-hybrid system, which showed that BplMYB46 could form homodimers and heterodimers with BplMYB6, BplMYB8, BplMYB11, BplMYB12, and BplMYB13. Relative beta-glucuronidase activity and chromatin immunoprecipitation assays showed that the interaction between BplMYB46 and the five MYBs increased the binding of BplMYB46 to the MYBCORE motif. A subcellular localization study showed that these MYBs were all located in the nucleus. Real-time fluorescence quantitative PCR results indicated that the expressions of BplMYB46 and the five $M Y B$ genes could be induced by salt and osmotic stress, and the BplMYB46 and BplMYB13 exhibited the most similar expression patterns. BplMYB46 and BplMYB13 co-overexpression in tobacco using transient transformation technology improved tobacco's tolerance to salt and osmotic stresses compared with overexpressing BplMYB13 or BplMYB46 alone. Taken together, these results demonstrated that BplMYB46 could interact with five other MYBs to form heterodimers that activate the transcription of target genes via an enhanced binding ability to the MYBCORE motif to mediate reactive oxygen species scavenging in response to salt and osmotic stresses.
\end{abstract}

Keywords: Betula platyphylla; MYB; dimerization; yeast two-hybrid; stress response

\section{Introduction}

The MYB family is one of the largest transcription factors (TFs) families in plants. MYBs have functions in various biological processes, such as regulating flavonoid biosynthesis, controlling cell differentiation, responding to hormone stimulus, mediating the cell wall biosynthesis, and enhancing or reducing biotic and abiotic stress tolerance of plants. The overexpression of MYB113 or MYB114 increased anthocyanin pigment levels in Arabidopsis [1]. AtMYB23 plays a role in the root epidermal cell type patterning in Arabidopsis [2]. An $M Y B$ gene, $M Y B H$, enhanced hypocotyl elongation in Arabidopsis by promoting auxin accumulation [3]. A series of studies demonstrated that AtMYB46 is a switch gene in the regulation of secondary wall deposition [4-9]. Genomic and transcriptomic analyses demonstrated that GmMYB (Glycine max) is related to resistance to Phakopsora pachyrhizi infection [10]. Overexpression of GmMYBJ1 increased tolerance to drought and cold stresses in soybean [11]. 
MYB proteins share a conserved MYB DNA-binding domain that binds to cis-acting elements of transcription factor genes and a diverse C-terminal modulator region to regulate the protein's activity. The DNA-binding domain and modulator region in MYBs are critical for protein-protein interactions, which can jointly regulate the growth and development of plants [12]. DmMYB (Drosophila melanogaster) interacts with CREB-binding proteins (CBPs), which promotes efficient progression through mitosis [13]. B-MYB forms a complex with itself to influence its transcriptional activity [14]. A study indicated that the MYB proteins interact with R/B-like bHLH proteins, jointly controlling the phenylpropanoid biosynthetic pathways, epidermal cell differentiation, and cell patterning in root hair or trichome development [15]. AtMYB44 interacts with regulatory components of ABA receptor 1 (RCAR1)/PYR1-like protein 9 (PYL9) as an abscisic acid (ABA) receptor in Arabidopsis, and negatively regulates ABA signaling [16]. The interaction between of MYB75 and KNOTTED ARABIDOPSIS THALIANA7 (KNAT7, a KNOX family protein) can regulate secondary cell wall biosynthesis [17]. In a recent study, bimolecular fluorescence complementation (BiFC) assay showed that MYB5 and MYB14 physically interact and play synergistic roles in inducing proanthocyanidin (PA) accumulation in Medicago truncatula [18]. GhJAZ2 (jasmonate zim-domain protein 2) can negatively regulate cotton fiber initiation via interacting with the R2R3-MYB transcription factor GhMYB25-like [19]. However, the interactions among MYB proteins or between MYBs and other proteins have rarely been studied. Such studies are important to illustrate the functions of these proteins in the growth and development of plants.

A previous study found that BplMYB46, an MYB gene from Betula Platyphylla (northeast white birch in China), enhances tolerance to salt and osmotic stresses when overexpressed in transgenic plants [20]. In the present study, we further studied the functions and interactions of BplMYB46. The yeast two-hybrid system demonstrated that BplMYB46 could form homodimers or heterodimers. Beta-glucuronidase (GUS) activity and chromatin immunoprecipitation (ChIP) assays further demonstrated that BplMYB46 could interact with BplMYB6, BplMYB8, BplMYB11, BplMYB12, and BplMYB13. Quantitative real-time reverse transcription PCR (qRT-PCR) indicated that BplMYB46 and BplMYB13 have similar expression patterns under salt and osmotic stresses. Co-overexpressing BplMYB13 and BplMYB46 improved the tolerance of transgenic tobacco to stress by enhancing the expression of their target genes. Our study provides insights into the important role of the interaction of BplMYB46 with other proteins in response to various stresses in plants.

\section{Results and Discussion}

\subsection{Sequence and Phylogenetic Analyses of Eight MYBs}

The cDNA sequences of eight new MYB transcription factors were obtained from the birch transcriptome (GenBank accession numbers: MK512591-MK512598). These MYBs encode proteins ranging from 240 to 341 amino acids (aa), with predicted molecular weights of 28.9 to $38.8 \mathrm{kDa}$ and pI values from 5.38 to 8.66 (Table S1). Domain prediction showed that these eight MYBs do not have transmembrane domains. At the amino acid sequence level, the coding regions of these MYBs shared 41 to $70 \%$ sequence identity. Multiple sequence alignments of the eight MYB proteins with BplMYB46 protein from northeast white birch in China were performed to examine the structures of the MYB transcription factors (Figure S1). BplMYB46 shared two conserved N-terminal DNA-binding $R$ regions with the eight MYBs, showing that they belong to the R2R3-MYB family of transcription factors. The evolutionary relationships of eight MYB proteins in B. platyphylla and all MYB proteins in Arabidopsis thaliana were analyzed based on an NJ phylogenetic tree (Figure S2). The results showed that the eight MYB proteins are close to Arabidopsis MYB proteins related to stress tolerance, xylem formation and pollen development in plants [21-25], suggesting the eight MYB proteins may have similar functions. 


\subsection{Analysis of the Dimerization of BplMYB46}

The yeast two-hybrid ( $\mathrm{Y} 2 \mathrm{H})$ system is an effective method to study protein-protein interactions $[15,26,27]$. In the present study, the interactions of BplMYB46 with the eight MYBs were analyzed using $\mathrm{Y} 2 \mathrm{H}$. First, when the eight pGBKT7-MYBs were used as baits and pGADT7-Rec-BplMYB46 was used as the prey, yeast expressing BplMYB6, BplMYB8, BplMYB11, BplMYB12, and BplMYB13 could grow on QDO/X- $\alpha$-Gal medium (Figure 1A), indicating that these five MYBs might interact with BplMYB46. When pGBKT7-BplMYB46-N (lacking the activation region) was used as the bait and the eight pGBKT7-MYBs were used as preys, BplMYB46, BplMYB6, BplMYB8, BplMYB11, BplMYB12 and BplMYB13 could grow on QDO/X- $\alpha$-Gal medium (Figure 1B), further illustrating that BplMYB46 can form homodimers with itself and heterodimers with the five MYBs.

A

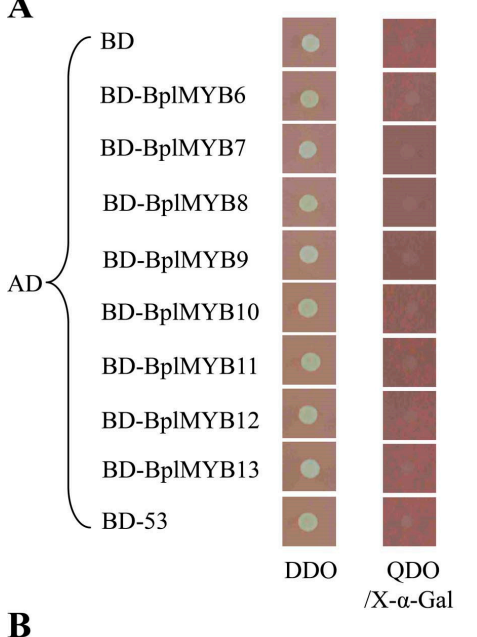

B

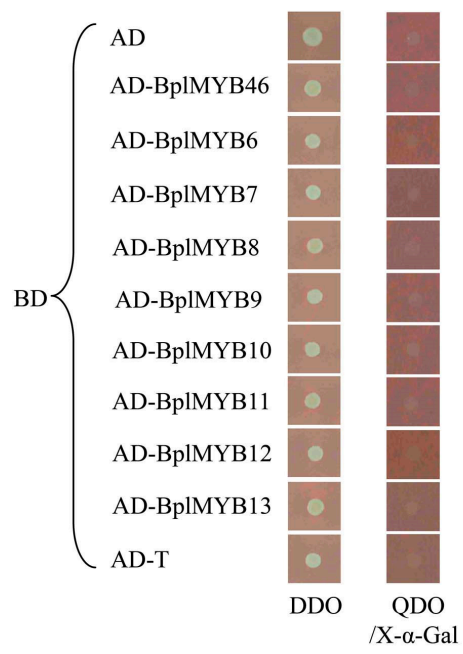

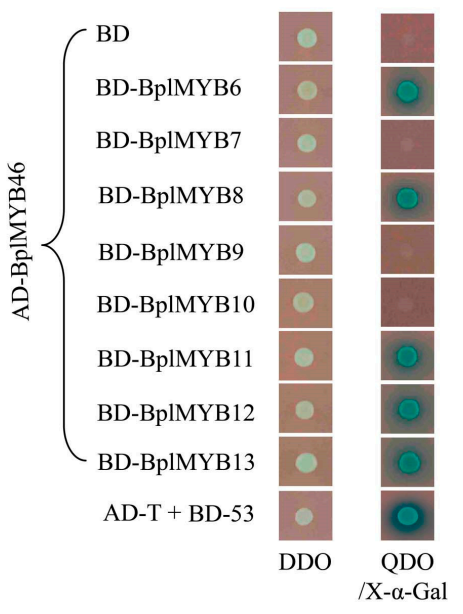

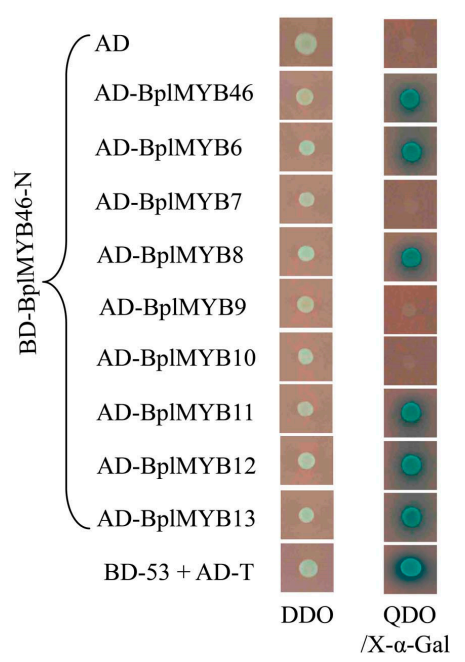

Figure 1. Homodimeric and heterodimeric analysis of BplMYBs using a yeast two-hybrid assay (Y2H). (A) BplMYB46 was cloned into a pGADT7-Rec vector (AD-prey) and interacted separately with the eight MYB proteins, which were fused to the GAL4 DNA binding domain in the yeast pGBKT7 vector (BD-baits). Empty AD-prey and each BD-bait were used as negative controls; AD-BplMYB46-prey and empty BD-bait were used as negative controls; and AD-T-prey and BD-53-bait were used as positive controls. (B) BplMYB46-N (without its activation regions) was cloned into pGBKT7 vector (bait) and interacted with itself and with the other eight MYB proteins cloned into the pGADT7-Rec vector (preys). Empty BD-bait and each AD-prey were used as negative controls; BD-BplMYB46-N-bait and empty AD-prey were used as negative controls; BD-53-bait and AD-T-prey were used as positive controls. The yeast cells were grown on SD/-Trp/-Leu (double dropout (DDO)) and selective dropout media: $\mathrm{SD} /$-Trp/-Leu/-His/-Ade/X- $\alpha$-Gal (quadruple dropout (QDO)/X- $\alpha$-Gal). 


\subsection{Verification by Transient Expression Assays}

To verify the above interactions identified by Y2H, BplMYB6, BplMYB8, BplMYB11, BplMYB12, and BplMYB13 were used for further analysis. The pROK2-BplMYB construct was used as an effector, and the fused MYBCORE (CAGTTA)-minimal 35S promoter-GUS was used as the reporter (Figure 2A). Equal ratios of pROK2-BplMYB46 and the five other pROK2-BplMYB were used as double-effectors, respectively. To eliminate the effect of activation by BplMYB46-BplMYB46 homodimers and the combination of BplMYB46 with one of the MYBs, which do not heterodimerize in yeast, the pROK2-BplMYB46 effector and pROK2-BplMYB46:pROK2-BplMYB7, as a double-effector, were used as controls, respectively. The effector and a reporter were co-transformed into tobacco leaves using the particle bombardment method (Figure 2B). The relative GUS activity of interaction of BplMYB46 and BplMYB6, BplMYB8, BplMYB11, BplMYB12, and BplMYB13 via the MYBCORE motif was significantly higher than that of only BplMYB46. Among them, the relative GUS activity of the interaction between BplMYB46 and BplMYB13 to MYBCORE was the highest. However, the relative GUS activity of BplMYB46:BplMYB7 bound to MYBCORE was similar to that of BplMYB46 only. Some studies indicated that a high level of relative GUS activity reflected a strong binding ability of proteins and specific target motifs [26-28]. Thus, our GUS results suggested that the combination of BplMYB46 and the five MYBs could enhance the binding ability to the MYBCORE motif.

A
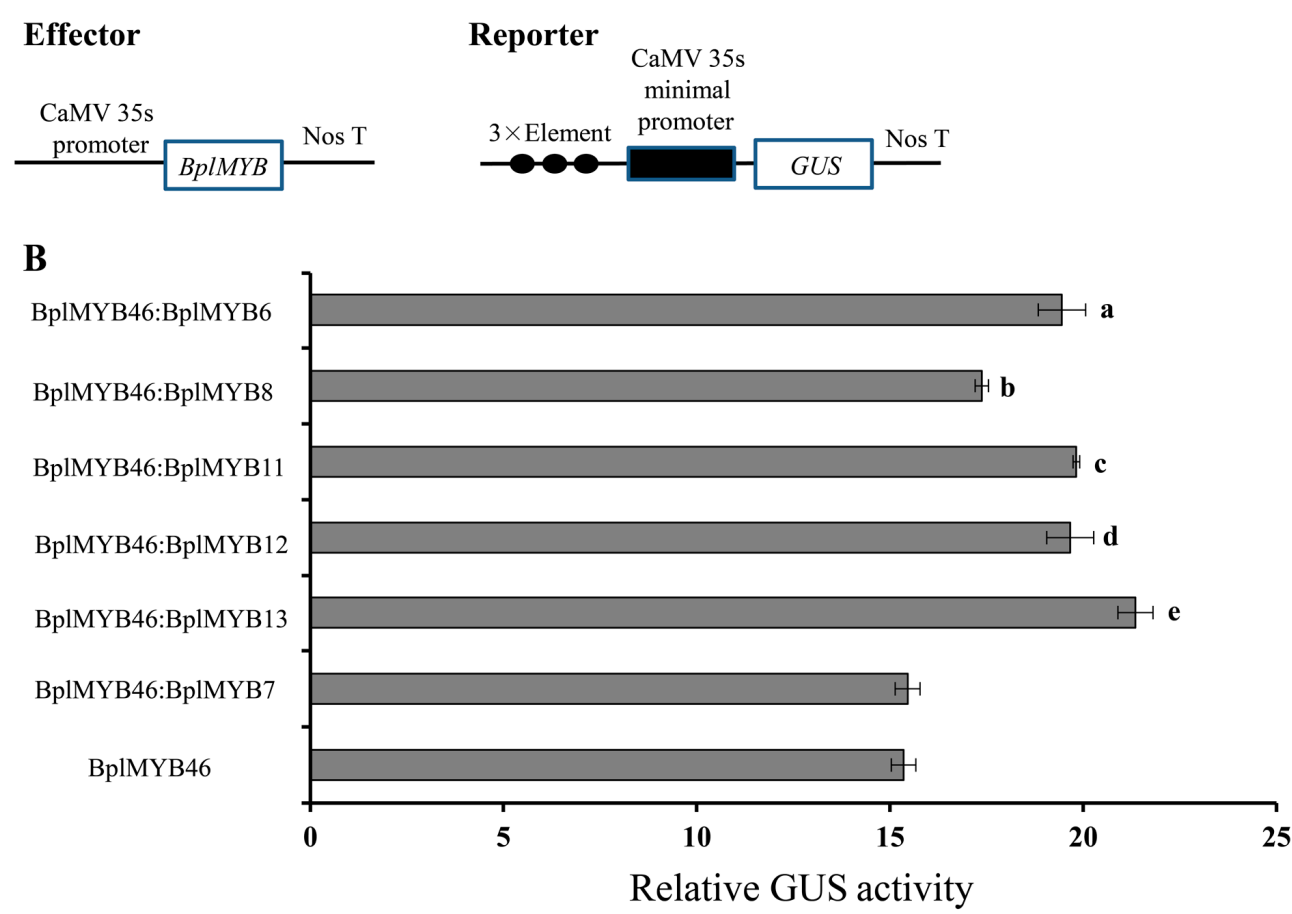

Figure 2. Beta-glucuronidase (GUS) activity analysis of the binding of BplMYB46 and five other MYBs to the MYBCORE sequence. (A) Schematic diagram of the effector and reporter constructs used in the GUS analysis. Triple tandem copies of the MYBCORE were fused with the 35S CaMV-46 minimal promoter and cloned into pCAMBIA1301 to drive expression of the GUS gene as the reporter construct. The coding sequences of BplMYBs were cloned into pROK2 under the control of the 35S promoter as the effector constructs. (B) The GUS activity assay results. Each effector and the reporter constructs were co-transformed into tobacco leaves. The combination of BplMYB46 with BplMYB7, which do not heterodimerize in yeast, was used as a control. The empty pROK2 vector was used as a negative control. The 35S-luciferase construct was transformed together with the reporter and effector into leaves to normalize the transformation efficiency. The GUS activity resulting from using the empty pROK2 vector as the effector was set to 1 . The error bars are the standard deviations, which were calculated from three independent biological repeats. Lower case letters indicate $p<0.05$. 


\subsection{ChIP Analysis}

ChIP analysis was used to determine the binding ability of BplMYB46 and the five MYBs to the MYBCORE motif (Figure 3). The binding abilities of the interactions of the BplMYB46-GFP protein and other five MYBs-GFP proteins to the MYBCORE motif were analyzed using real-time PCR. The results indicated that the enrichment of the MYBCORE motif under the interaction of BplMYB46 with the other MYBs was approximately 2.5-4 fold higher than the enrichment achieved from the MYB46 promoter. The enrichment of the MYBCORE motif in response to the BplMYB46 protein alone was approximately 1.5 folds higher than the enrichment of the BplMYB46 promoter. Thus, the binding abilities of BplMYB46-GFP when interacting with the other five MYBs-GFP proteins to the MYBCORE motif were stronger than the binding between BplMYB46-GFP and the MYBCORE motif. Our results demonstrated that the interaction between BpIMYB46 and the other five MYBs enhanced their binding to the MYBCORE motif.

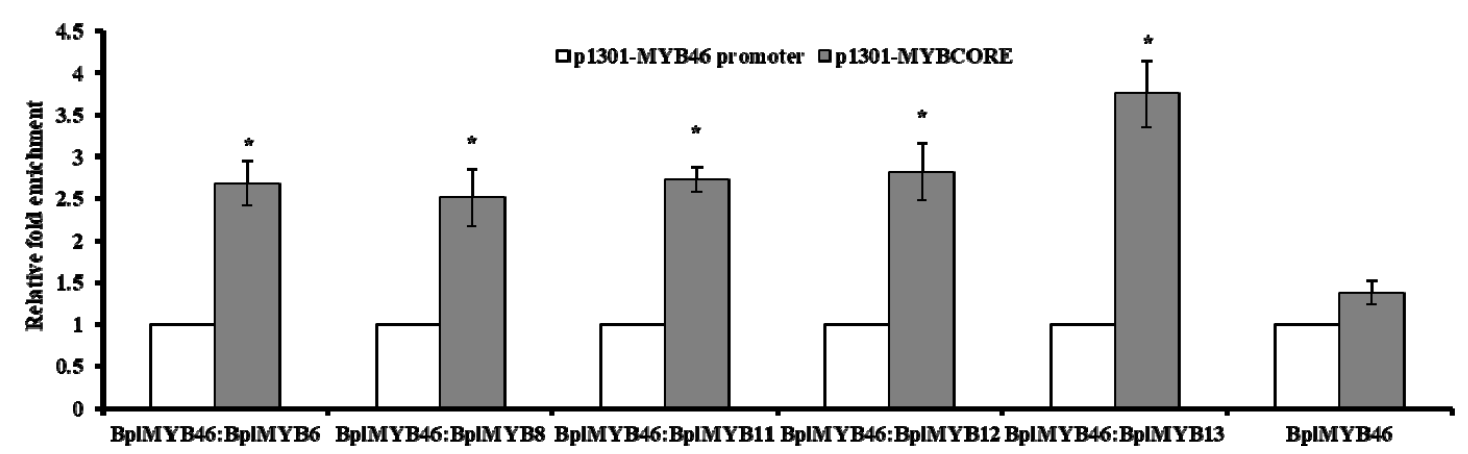

Figure 3. Chromatin immunoprecipitation (ChIP) analysis of BplMYB46 and five other MYBs binding to the MYBCORE motif. Quantitative real-time PCR analysis showing the enrichment of the promoter sequences, including the MYBCORE motif after chromatin immunoprecipitation. The values for the enrichment of the BplMYB46 promoter sequences were set to 1. Chromatin from whole seedlings was isolated from pBI121-BPIMYB46-GFP and pBI121-MYB-GFP birch plants produced by Agrobacterium tumefaciens-mediated transient transformation. The error bars indicate the standard deviation (SD) from three biological replicates. ${ }^{*}$ indicates a significant difference $(p<0.05)$.

\subsection{Subcellular Localization of BplMYB46, 6, 8, 11, 12, and 13}

To study the subcellular localization of the MYBs, the MYB-GFP fusion genes and the GFP gene were separately transformed into onion epidermal cells using the particle bombardment method. GFP alone was distributed throughout the transformed cells, whereas the six MYB-GFP fusion proteins were exclusively localized to the nucleus (Figure 4), which suggested that BplMYB46 and the other five MYBs are nuclear proteins. 


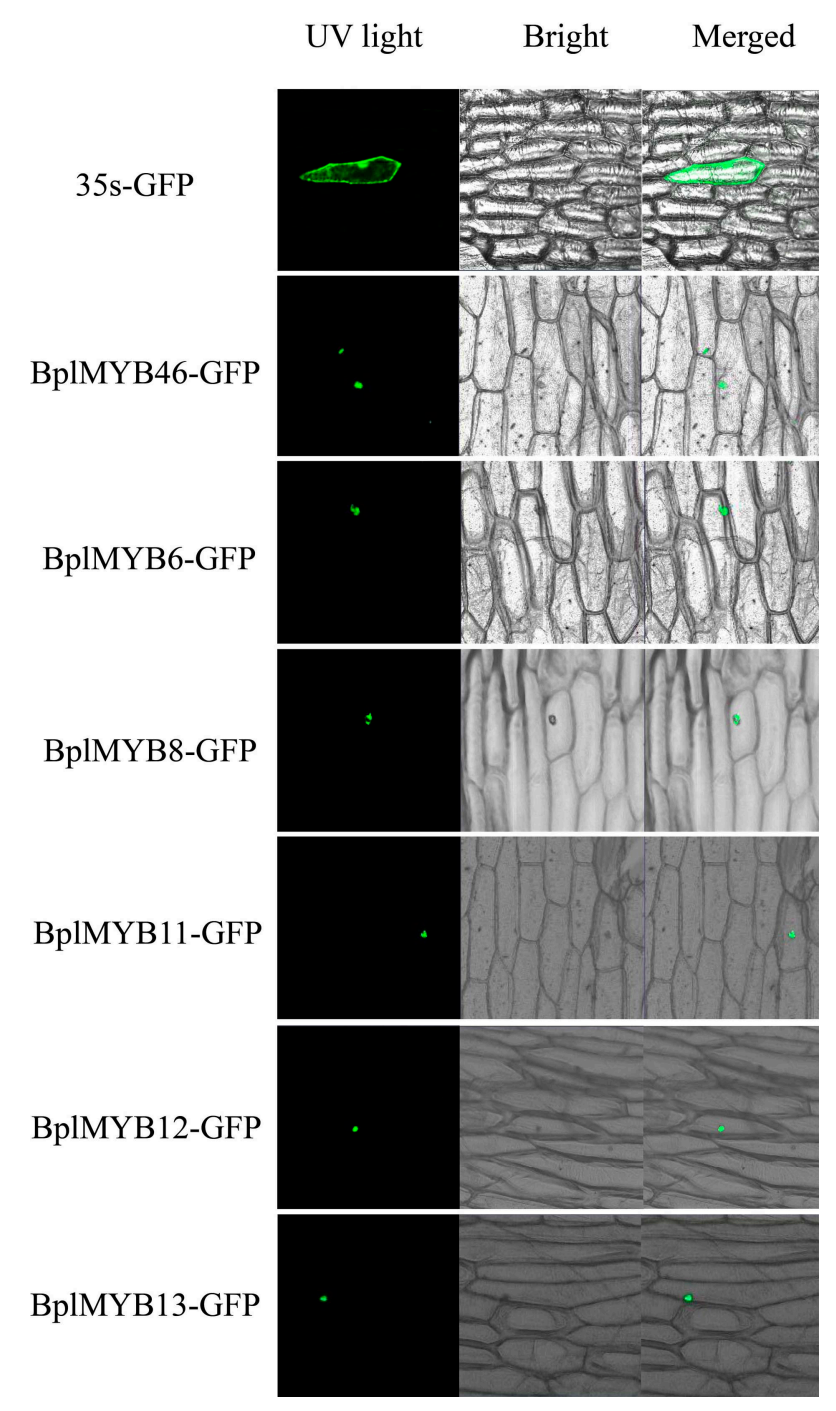

Figure 4. Nuclear localization of BplMYB proteins. The BplMYB-GFP fusion genes and GFP (control) were transiently expressed in onion epidermal cells using the particle bombardment method. The transformed cells were cultured on Murashige-Skoog (MS) medium for 24-48 h and visualized using a confocal microscope at $488 \mathrm{~nm}$.

2.6. Expression Patterns of BplMYB46, 6, 8, 11, 12, and 13 in Response to Abiotic Stresses

To investigate the expression patterns of BplMYB46, 6, 8, 11, 12, and 13 in response to salt and osmotic treatments, a qRT-PCR analysis was performed (Figure 5). Under salt stress, the expression levels of BplMYB46, 6, 8, 11, 12, and 13 gradually increased at 6, 12, and $24 \mathrm{~h}$, reaching a peak level at $24 \mathrm{~h}$ and then rapidly decreased at $48 \mathrm{~h}$. Interestingly, the expression patterns of BplMYB46, 12, and 13 were similar, and their expression levels were higher than those of BplMYB6, 8, and 11 at $24 \mathrm{~h}$ (Figure 5A). Under osmotic stress, the expression analysis showed that the expression patterns of these genes were clustered into two types (Figure 5B). In the first type, the expression levels of BplMYB46, 6, 8,12 , and 13 increased gradually at 6 and $12 \mathrm{~h}$, reaching a peak level at $12 \mathrm{~h}$; thereafter, their levels decreased. Interestingly, the expression pattern of BplMYB46 was more similar to that of BplMYB13 than to that of BplMYB6, 8, and 12. In the second type, the expression of BplMYB11 gradually increased at 6,12 , and $24 \mathrm{~h}$, reaching a peak level at $24 \mathrm{~h}$, and then rapidly decreasing at $48 \mathrm{~h}$. These results showed that the expressions of BplMYB46 and the $5 M Y B$ genes could be induced by salt or osmotic stress. In addition, we found that BplMYB46 and BplMYB13 shared very similar expression patterns under salt or osmotic stress. 
A
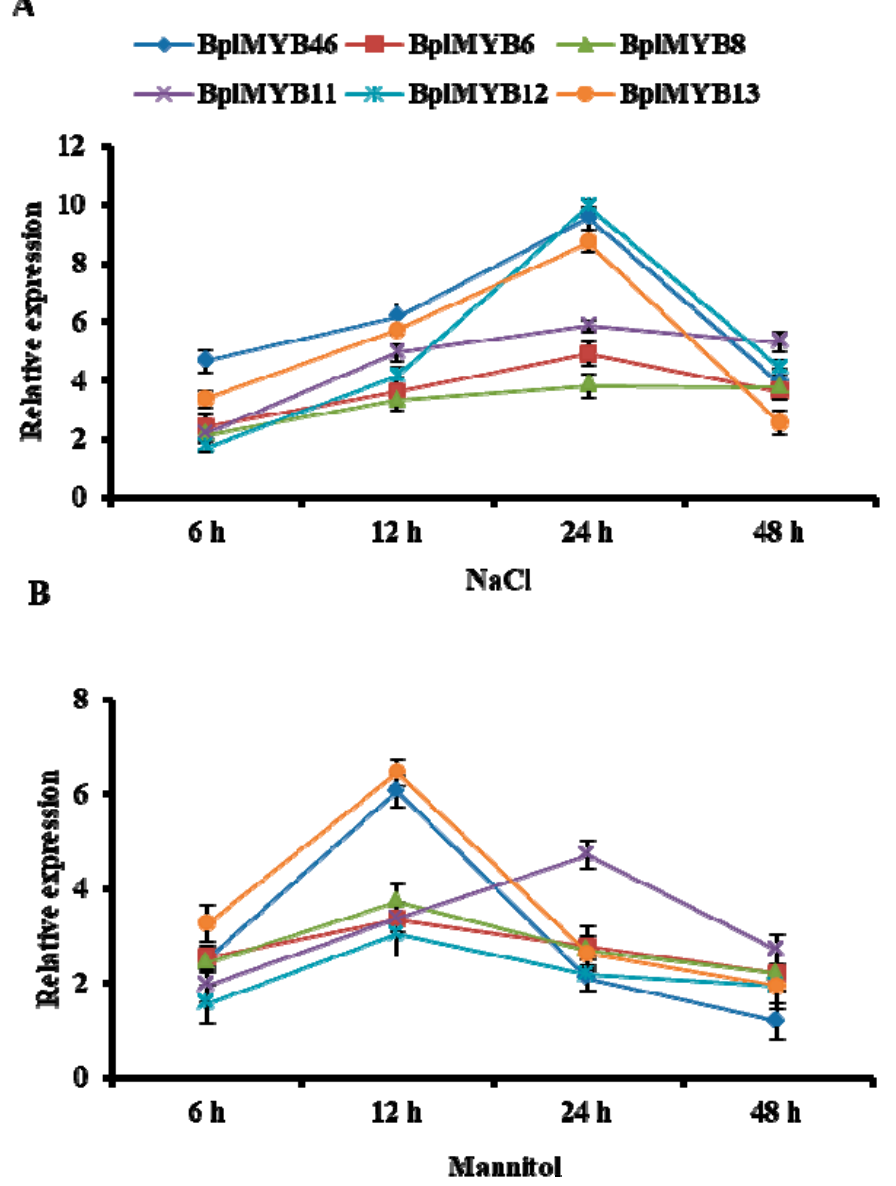

Figure 5. Expression profiles of BplMYB46, BplMYB6, BplMYB8, BplMYB11, BplMYB12, and BplMYB13 under different abiotic stresses. Two-month-old birch seedlings were treated with $200 \mathrm{mM} \mathrm{NaCl}$ (A) or $200 \mathrm{mM}$ Mannitol (B) for different times. Fresh-watered plants were grown in parallel as controls. After these treatments, the stems of the seedlings from each sample were harvested and pooled for real-time reverse transcription (RT)-PCR analyses. The error bars indicate the standard deviation (SD) from three biological replicates.

2.7. Plants Overexpressing BplMYB46 and BplMYB13 Display Alleviated Oxidative Stress and Diminished Cell Membrane Damage

Our study showed that BplMYB46 could interact with BplMYB6, 8, 11, 12, and 13, and the expression pattern of BplMYB46 was very similar to that of BplMYB13 under salt or osmotic stress. Therefore, BplMYB13 was selected for further study. We tested whether co-overexpression of BplMYB46 and BplMYB13 could improve abiotic stress tolerance compared with overexpression of BplMYB13 or BplMYB46, separately. In this experiment, the leaves of BplMYB46-BplMYB13 co-overexpressing plants, BplMYB13 overexpressing plants, BplMYB46 overexpressing plants, and WT (wild-type) tobacco plants were stained using nitroblue tetrazolium (NBT), 3,3'-diaminobenzidine (DAB), and Evans blue under normal conditions, and under salt and osmotic stresses. NBT and DAB staining reveal the cellular levels of $\mathrm{O}_{2}{ }^{-}$and $\mathrm{H}_{2} \mathrm{O}_{2}$, two of the main ROS species involved in stress signaling and oxidative injuries [29]. Under control conditions, no obvious difference in NBT and DAB staining was observed among BplMYB46 and BplMYB13 co-overexpressing plants, BplMYB13 or BplMYB46 only overexpressing plants, and WT tobacco. By contrast, under $\mathrm{NaCl}$ or mannitol treatments at $6 \mathrm{~h}$, the leaves of BplMYB46 and BplMYB13 co-overexpressing plants exhibited less blue and brown staining compared with those from plants overexpressing BplMYB13 or BplMYB46 alone and WT plants (Figure 6A,B). Evans blue staining can reflect cell death in plants, as manifested by damage to the cell membrane [30]. The results showed that the BplMYB46 and BplMYB13 co-overexpressing transgenic plants displayed less intense 
blue staining compared with plants overexpressing BplMYB13 or BplMYB46 alone or WT plants under salt and osmotic stress conditions (Figure 6C). These results showed that BplMYB46 and BplMYB13 co-overexpressing transgenic plants had enhanced abilities to scavenge ROS and had decreased levels of cell death, demonstrating that the interaction between BplMYB46 and BplMYB13 could increase plant stress resistance.
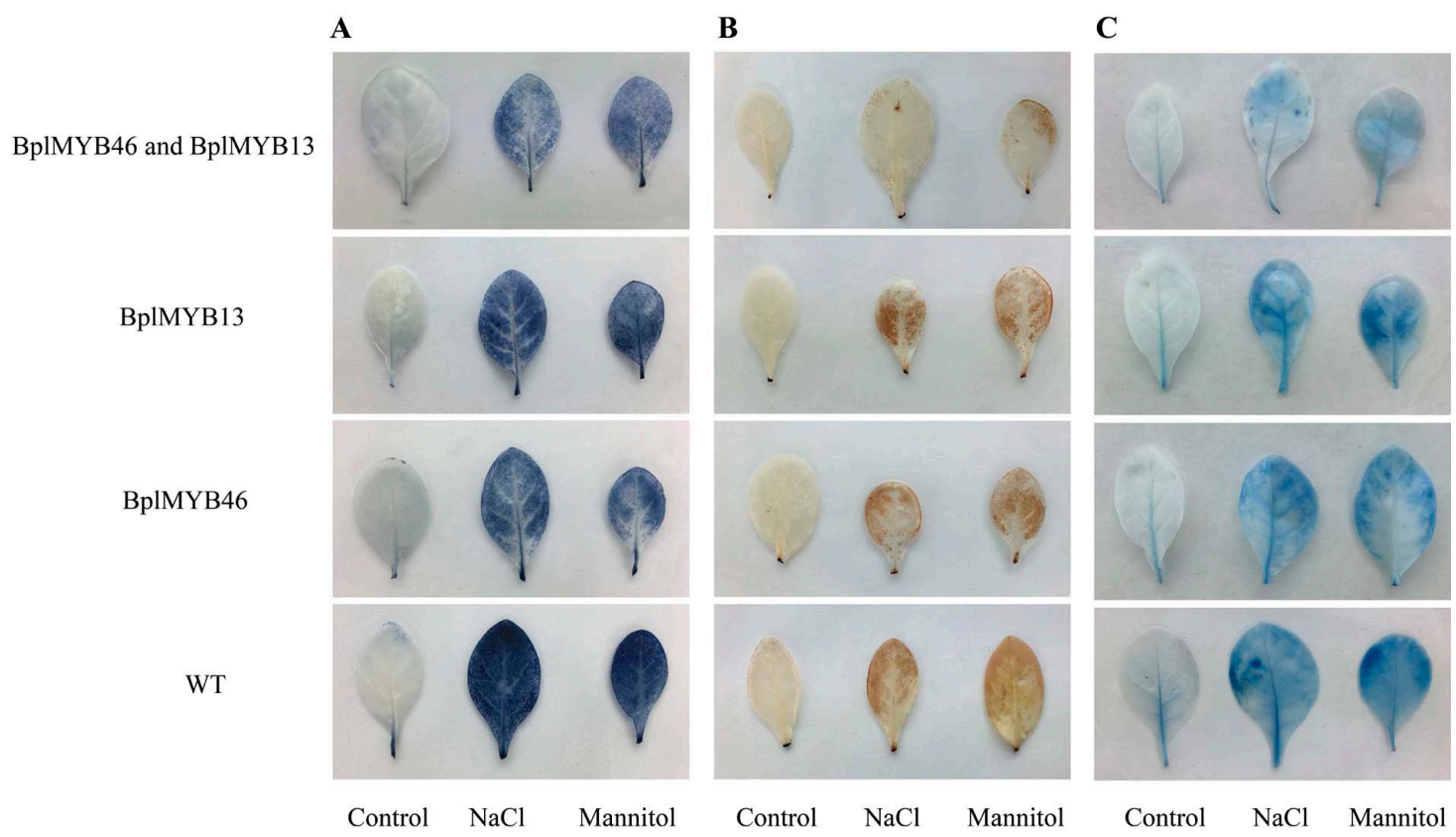

Figure 6. The reactive oxygen species (ROS) levels and cell death in BplMYB46 and BplMYB13 transgenic and wild-type tobacco under $\mathrm{NaCl}$ and Mannitol treatment. (A, B) Cellular levels of $\mathrm{O}_{2}{ }^{-}$and $\mathrm{H}_{2} \mathrm{O}_{2}$ in BplMYB46 or BplMYB13-transformed and wild-type (WT) plants under normal conditions, and under salt or drought stress. Leaves from BplMYB13-transformed and WT plants were pretreated with of $\mathrm{NaCl}$ or mannitol, and the levels of $\mathrm{O}_{2}{ }^{-}$and $\mathrm{H}_{2} \mathrm{O}_{2}$ were visualized using nitroblue tetrazolium (NBT) and 3,3'-diaminobenzidine (DAB) staining, respectively. (C) Evans Blue staining analysis of cell death. The leaves sampled from two-month-old transgenic and WT plants were treated with $\mathrm{NaCl}$ or mannitol at $6 \mathrm{~h}$ and used for histochemical staining, treatment with water only was used as a control. Each experiment was repeated at least three times, and approximately 10 leaves harvested from multiple seedlings were inspected.

\subsection{Physiological Characterization of BplMYB46 and BplMYB13 Co-Overexpressing Plants}

Superoxide dismutase (SOD), peroxidase (POD), and glutathione-S-transferase (GST) activities, $\mathrm{H}_{2} \mathrm{O}_{2}$ and malondialdehyde (MDA) contents, and electrolyte leakage have been used to analyze stress tolerance associated with TFs in plants [31,32]. In this study, the activities of SOD, POD, and GST, the $\mathrm{H}_{2} \mathrm{O}_{2}$ and MDA contents, and electrolyte leakage were used to detect the resistance of plants co-overexpressing BplMYB46 and BplMYB13 to salt and osmotic stresses; water was used the control (Figure 7). Under control conditions, the activities of SOD, POD, and GST in BplMYB46 and BplMYB13 co-overexpressing, BplMYB13 or BplMYB46 only overexpressing plants, and WT plants showed almost no differences. However, under salt and osmotic stresses at $6 \mathrm{~h}$, the activities of SOD, POD, and GST in the BplMYB46 and BplMYB13 co-overexpressing plants were significantly higher compared with those in BplMYB13 or BplMYB46 only overexpressing plants, and WT plants (Figure 7A-C). Under control conditions, there was hardly any difference in the $\mathrm{H}_{2} \mathrm{O}_{2}$ and MDA contents between the BplMYB46 and BplMYB13 co-overexpressing plants, the BplMYB13 or BplMYB46 only overexpressing plants, and the WT plants. However, under salt and osmotic stresses at $6 \mathrm{~h}$, in the BplMYB46 and BplMYB13 co-overexpressing plants, the $\mathrm{H}_{2} \mathrm{O}_{2}$ and MDA contents were significantly lower than those in the BplMYB13 or BplMYB46 only overexpressing plants and WT plants (Figure 7D,E). The $\mathrm{H}_{2} \mathrm{O}_{2}$ and MDA 
contents (levels of $\mathrm{O}_{2}^{-}$) can negatively reflect the ROS scavenging ability of plants; therefore, our results demonstrated that the interaction between BplMYB46 and BplMYB13 could enhance the plants' ROS scavenging ability. Electrolyte leakage has been used as a measure of cell death. Under control conditions, there was no significant difference among the BplMYB46 and BplMYB13 co-overexpressing plants, the BplMYB46 overexpressing plants, and WT plants. However, under salt and osmotic stresses at $6 \mathrm{~h}$, the BplMYB46 and BplMYB13 co-overexpressing transgenic plants had significantly lower levels of electrolyte leakage than those in the BplMYB46 overexpressing plants and WT plants, indicating that the combination with BplMYB13 could decrease cell death (Figure 7F). These results suggested that the interaction of BplMYB46 with BplMYB13 could enhance tolerance to salt or osmotic stresses by enhancing the ROS scavenging ability and decreasing cell death in plants.

A

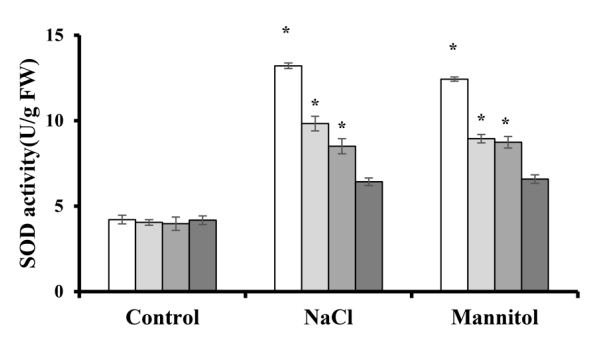

C
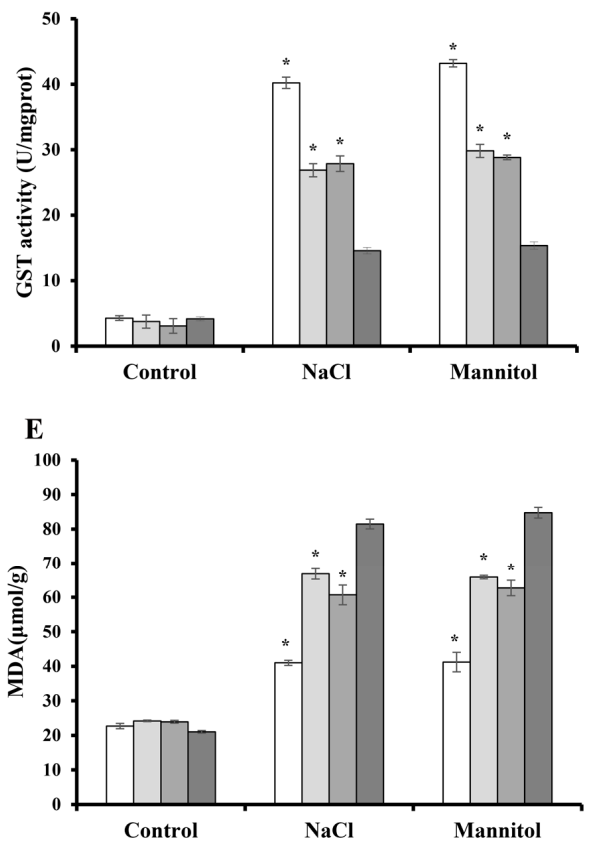

B

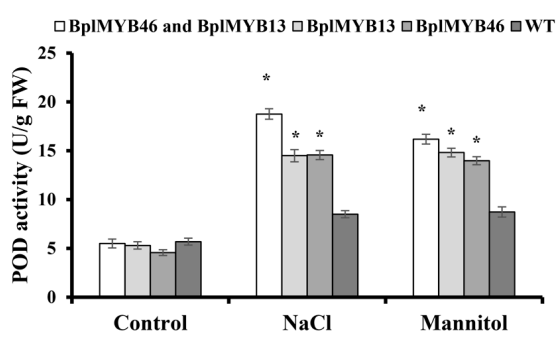

D

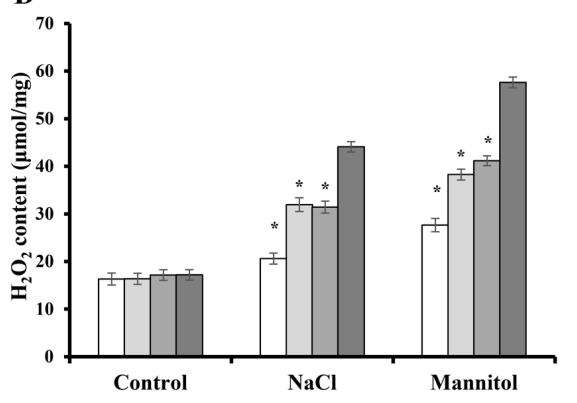

F

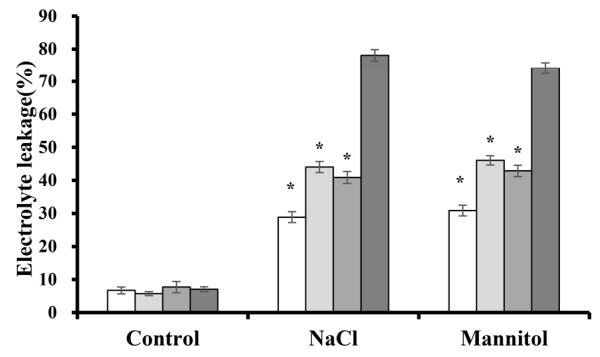

Figure 7. Salt and osmotic stress tolerance analyses of BplMYB46 and BplMYB13 transgenic and wild-type (WT) tobacco. Superoxide dismutase (SOD) activity (A), peroxidase (POD) activity (B), glutathione-S-transferase (GST) activity (C), and $\mathrm{H}_{2} \mathrm{O}_{2}$ contents (D), malondialdehyde (MDA) contents (E), and electrolyte leakage (F) of transgenic and WT tobacco under salt and osmotic stress. The seedlings sampled from the transgenic tobacco using transient Agrobacterium-mediated transformation and WT plants were treated with $\mathrm{NaCl}$ and mannitol at $6 \mathrm{~h}$, treatment with water used as a control. Each experiment was repeated at least three times, and approximately 10 leaves harvested from multiple seedlings were inspected. The error bars indicate the standard deviation (SD) from three biological replicates. ${ }^{*}$ indicates a significant difference $(p<0.05)$.

\subsection{The Relative Expression of Target Genes}

To further analyze whether the interaction of BplMYB46 with BplMYB13 could affect the expression of target genes, the relative expression levels of SOD, POD, and GST in BplMYB46 and 
BplMYB13 co-overexpressing plants, BplMYB46 or BplMYB13 only overexpressing plants, and WT plants under salt and osmotic stresses were examined. Under salt or osmotic treatment, the relative expression levels of $S O D, P O D$, and GST genes were significantly higher in the BplMYB46 and BplMYB13 co-overexpressing plants than in the BplMYB46 or BplMYB13 only overexpressing plants and WT plants compared with those under fresh water treatment (Figure 8). Analysis of the DNA sequences on the PLACE website showed that the MYBCORE motif exists in the promoters of the five genes. Thus, our results suggested that the interaction of BplMYB46 with BplMYB13 might enhance the expression levels of $S O D, P O D$, and GST genes by binding to the MYBCORE cis-acting element.

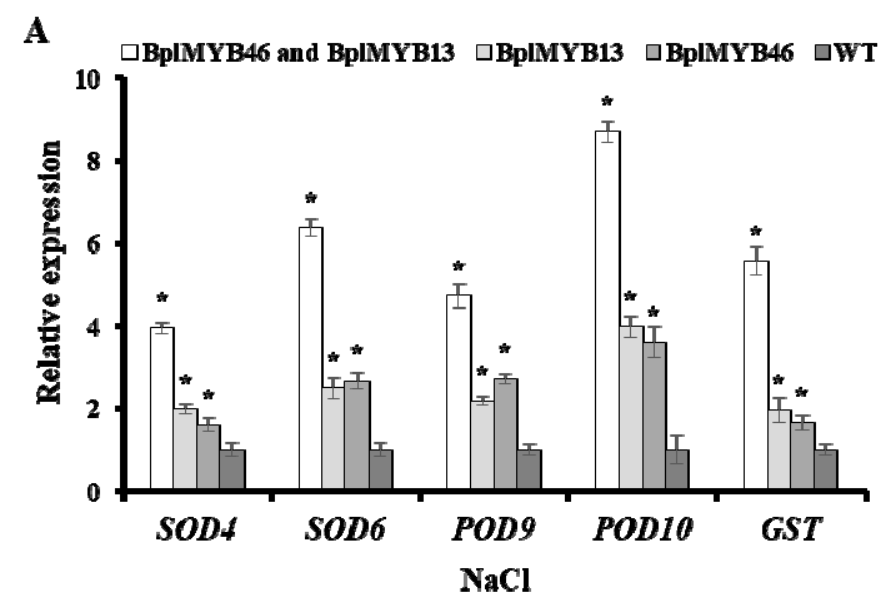

B

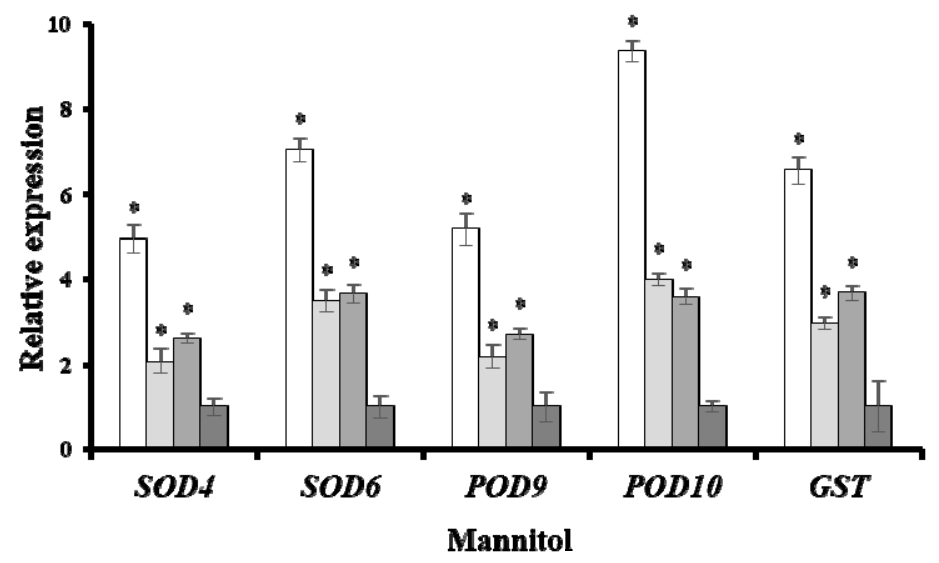

Figure 8. The relative expression analyses of target genes in BplMYB46 and BplMYB13 transgenic and wild-type (WT) birch. The relative expression levels of superoxide dismutase (SOD), peroxidase (POD) and glutathione-S-transferase (GST) genes of transgenic and WT tobacco under salt stress (A) under osmotic stress (B). The seedlings sampled from the transgenic tobacco using transient Agrobacterium-mediated transformation and WT plants were treated with $\mathrm{NaCl}$ and mannitol at $6 \mathrm{~h}$, treatment with water only was used as a control. The error bars indicate the standard deviation (SD) from three biological replicates. ${ }^{*}$ indicates a significant difference $(p<0.05)$.

\section{Materials and Methods}

\subsection{Plant Materials and Growth Conditions}

Tobacco seedlings were planted on half Murashige-Skoog (1/2 MS) medium in a growth chamber with a $16 / 8 \mathrm{~h} \mathrm{light/dark} \mathrm{cycle} \mathrm{and} \mathrm{an} \mathrm{average} \mathrm{temperature} \mathrm{of} 25^{\circ} \mathrm{C}$.

Northeast white birch seeds were planted in pots containing a mixture of perlite/soil (2:1) in a greenhouse under controlled conditions of $16 / 8 \mathrm{~h}$ light/dark, an average temperature of $25^{\circ} \mathrm{C}$, and $70-75 \%$ relative humidity. 


\subsection{Sequence Analysis of MYB Transcription Factors}

The cDNA sequences of eight MYB transcription factors were obtained from the birch transcriptome. The theoretical molecular weight (MW) and isoelectronic point (pI) predictions for each deduced MYB were calculated using the ExPASy compute pI/Mw tool (http:/ / www.expasy. org/tools/protparam.html). Transmembrane domains were predicted using the TMHMM server 2.0 (http://www.cbs.dtu.dk/services/TMHMM/). Multiple sequence alignments of BplMYB46 with the eight MYBs were performed using the ClustalW program (http://www.ebi.ac.uk/clustalw/). An unrooted phylogenetic tree was constructed using MEGA5.1, according to the neighbor-joining (NJ) method with 1000 bootstrap replicates. The sequences of the Arabidopsis MYB domain proteins were downloaded from the Arabidopsis genome TAIR 9.0 (http:/ / www.arabidopsis.org/).

\subsection{Heterodimer and Homodimer Assays for BplMYB46}

The open reading frame (ORF) of the BplMYB46 cDNA, without the terminal codon, was inserted into the pGADT7-Rec vector via its SmaI (Promega, Madison, WI, USA) site as the prey construct. The eight BplMYBs, without their terminal codons, were inserted into pGBKT7 and used as bait constructs. The pGBKT7 empty vector and pGADT7-Rec-BplMYB46, and pGBKT7 and pGADT7-Rec empty vectors were used as negative controls. Baits and preys were transformed into the yeast strain Y2HGold, and then plated on SD/-Trp/-Leu (double dropout (DDO)) and SD/-Trp/-Leu /-His/-Ade/X- $\alpha$-Gal (quadruple dropout (QDO) media at $30{ }^{\circ} \mathrm{C}$ for $3-5$ days.

A truncated fragment of the BplMYB46 cDNA, lacking the region encoding the transactivation region, was constructed into the pGBKT7 vector as the bait, and BplMYB46 and the eight MYBs lacking their terminal codons were constructed into the pGADT7-Rec as preys. Construct pGBKT7-BplMYB46 and pGADT7-Rec empty vector, and pGBKT7 and pGADT7-Rec empty vectors were used negative controls. Vector pGBKT7-53 and pGADT7-Rec-T were used positive controls. Vector pGBKT7-53 encodes the Gal4 DNA-BD fused with murine p53; pGADT7-T encodes the Gal4 AD fused with SV40 large T-antigen. The p53 and large T-antigen are known to interact in a yeast two-hybrid assay; therefore, mating Y2HGold (pGBKT7-53) with Y187 (pGADT7-T) allows the cells to grow in QDO mediums $/ \mathrm{X}-\alpha-$ Gal. Baits and preys were transformed into $\mathrm{Y} 2 \mathrm{H}$ cells, and were grown on DDO and $\mathrm{QDO} / \mathrm{X}-\alpha$-Gal media at $30^{\circ} \mathrm{C}$ for $3-5$ days. All the primer sequences used are shown in Table S2.

\subsection{Transient Expression Assays}

The full length ORFs of BplMYB6, BplMYB7, BplMYB8, BplMYB11, BplMYB12, and BplMYB13 were inserted separately into vector pROK2. An equal proportion of pROK2-BplMYB46 and other pROK2-BplMYBs formed the double-effectors, respectively. The fused MYBCORE-minimal 35S CaMV promoter-GUS construct was used as the reporter. The effector and reporter vector were co-transformed into tobacco leaves using the particle bombardment method (Bio-Rad, Hercules, CA, USA). The effector of pROK2, pROK2-BplMYB46 and pROK2-BplMYB46: pROK2-BplMYB7 served as controls, respectively. The firefly luciferase gene driven by the CaMV $35 \mathrm{~S}$ promoter was also co-transformed as an internal control for normalization of the transformation efficiency. The GUS activity from the empty pROK2 vector as an effector was set to 1 . GUS and luciferase activities were determined according to previously published methods [33], and the data are shown as the averages of three biological replicates. The primer sequences are shown in Table S3.

\subsection{Chromatin Immunoprecipitation (ChIP) Analysis}

The ORFs of BplMYB6, BplMYB8, BplMYB11, BplMYB12, BplMYB13, and BplMYB46 without their terminal codons were inserted separately into vector pBI121, upstream of GFP, and the six pBI121-MYB-GFPs were transformed separately into EHA 105 Agrobacterium competent cells. pBI121-BplMYB and pBI121-BplMYB46 were co-transformed into birch by transient Agrobacterium-mediated transformation [34]. At $48 \mathrm{~h}$ after transformation, the transgenic plants 
were collected for the ChIP assay. DNA and protein were cross-linked using $1 \%$ formaldehyde, then the chromatin was sheared into 200-750 bp fragments using sonication, and $10 \%$ of the sample was saved as the input control. The remaining sonicated chromatin was divided into two parts, which were respectively added with anti-GFP antibodies (ChIP+) and no antibody (negative control, ChIP-). The DNA truncated fragments were released by reversing the cross-linking at $65{ }^{\circ} \mathrm{C}$ for $6 \mathrm{~h}$, and were then digested by proteinase $\mathrm{K}(0.2 \mathrm{mg} / \mathrm{mL})$ for $1 \mathrm{~h}$ at $45^{\circ} \mathrm{C}$ to remove any residual proteins. The immunoprecipitated DNA was purified using chloroform extraction. The enrichment of the truncated promoter, including the MYBCORE motif was determined on the chromatin DNA samples after immunoprecipitation, using real-time PCR. The values for the bound MYBCORE motif were normalized against those of the truncated BplMYB46 promoter. Three biological replications were used in this experiment. The primer sequences are shown in Table S4.

\subsection{Subcellular Localization Analysis}

The pBI121-BplMYBs-GFP fusion gene and pBI121-GFP (control) were transiently expressed in onion epidermal cells using the particle bombardment (Bio-Rad) method. The transformed cells were then cultured on MS medium for 24-48 h and analyzed using a confocal laser-scanning microscope at $488 \mathrm{~nm}$ (LSM410, Zeiss, Jena, Germany).

\subsection{Plant Stress Treatments and Real-Time Reverse Transcription (RT)-PCR}

Two-month-old birch seedlings were collected at the same time after treatment for 6, 12, 24, and $48 \mathrm{~h}$ under $200 \mathrm{mM} \mathrm{NaCl}$ (salt stress) or $200 \mathrm{mM}$ Mannitol. Mannitol is a naturally occurring sugar alcohol that can cause osmotic stress and has been widely used for studies of osmotic stress in plants. Seedlings watered with fresh water were used as controls. Total RNA was isolated from birch using the CTAB method and treated with DNase I (Takara Bio Inc., Shiga, Japan) and RNase free to remove DNA contamination. The total RNA was treated with DNase I and was reverse transcribed into cDNA using a PrimeScript ${ }^{\mathrm{TM}} \mathrm{RT}$ reagent Kit (Takara Bio Inc.) and real-time PCR was performed for BplMYB46, BplMYB6, BplMYB8, BplMYB11, BplMYB12, and BplMYB13. The sequences of the primers used are listed in Table S5. Real-time quantitative RT-PCR was performed with the SYBR Premix Ex Taq ${ }^{\mathrm{TM}}$ kit. DNA were amplified, according to the following procedure: $94{ }^{\circ} \mathrm{C}$ for $30 \mathrm{~s} ; 45$ cycles at $94{ }^{\circ} \mathrm{C}$ for $12 \mathrm{~s}, 58^{\circ} \mathrm{C}$ for $30 \mathrm{~s}$, and $72{ }^{\circ} \mathrm{C}$ for $45 \mathrm{~s}$; followed by $79^{\circ} \mathrm{C}, 1 \mathrm{~s}$ for plate reading. A melting curve was generated for each sample to assess the purity of the amplified products. The average values of the cycle thresholds $(\mathrm{Ct})$ of the $\alpha$-tubulin and ubiquitin genes were used as internal references. The relative expression ratios were calculated from the $\mathrm{Ct}$ values according to the delta-delta $\mathrm{Ct}$ method [35]. The relative transcription level was calculated as the transcription level under stress treatment divided by the transcription level under control conditions. Three independent biological replicates were performed in this experiment.

\subsection{Stress Tolerance Analysis of the Interaction of BplMYB46 with BplMYB13}

The overexpression constructs pROK2-BplMYB46 and pROK2-BplMYB13 were co-transformed, and pROK2-BplMYB13 and pROK2-BplMYB46 were separately transformed into tobacco using transient Agrobacterium-mediated transformation. The tobacco co-overexpressing BplMYB46 and BplMYB13, overexpressing BplMYB13 or BplMYB46 only, and the WT were treated with $200 \mathrm{mmol} / \mathrm{L}$ $\mathrm{NaCl}$ or $200 \mathrm{mmol} / \mathrm{L}$ Mannitol stresses for $6 \mathrm{~h}$; treatment with water only was used as a control. The detached leaves from tobacco were infiltrated with NBT $(1.0 \mathrm{mg} / \mathrm{mL})$ and DAB $(1.0 \mathrm{mg} / \mathrm{mL})$ following published procedures [29], and Evans blue $(1.0 \mathrm{mg} / \mathrm{mL})$ staining was performed to detect cell death following a previously published protocol [36]. SOD, $\mathrm{POD}, \mathrm{GST}, \mathrm{H}_{2} \mathrm{O}_{2}, \mathrm{MDA}$, and electrolyte leakage measurements were conducted according to previously published methods $[27,31]$. Three biological replicates were performed. 


\subsection{Expression Analysis of Target Genes}

Tobacco co-overexpressing BplMYB46 and BplMYB13, tobacco overexpressing BplMYB13 or BplMYB46 only, and WT tobacco were collected after $6 \mathrm{~h}$ of treatment with $200 \mathrm{mM} \mathrm{NaCl}$ or $200 \mathrm{mM}$ Mannitol, and seedlings watered with fresh water were used as controls. Three independent biological replicates were performed. Total RNA was extracted and treated with DNase I before being reverse transcribed into cDNA using a PrimeScript ${ }^{\mathrm{TM}} \mathrm{RT}$ reagent Kit (Takara). Real-time PCR was then performed for two SOD, two POD, and 1 GST (GenBank accession number: MK532397) genes. The sequences of the primers used are listed in Table S6.

\subsection{Statistical Analysis}

Analysis of variance (ANOVA) was used to analyze the relative GUS activity. All statistical analyses were performed using SPSS software (IBM Corp., Armonk, NY, USA), version 18.0.

\section{Conclusions}

Previously, we reported that BplMYB46 is involved in abiotic stress tolerance and secondary wall deposition. In the present study, we further showed that BplMYB46 could homodimerize with itself and heterodimerize with the BplMYB6, BplMYB8, BplMYB11, BplMYB12, and BplMYB13. The interaction of BplMYB46 and the five MYB proteins could improve the binding ability of BplMYB46 to the MYBCORE motif. In addition, BplMYB6, BplMYB8, BplMYB11, BplMYB12, and BplMYB13 exhibited similar expression patterns to that of BplMYB46 in birch under salt and osmotic treatment. A subcellular localization study showed that these MYBs were all targeted to the nucleus. BplMYB46 and BplMYB13 co-overexpression in tobacco improved the plants' tolerance to salt and osmotic stresses compared with BplMYB46 overexpression alone and further demonstrated that the interaction of BplMYB46 with BplMYB13 might increase plant stress tolerance.

Supplementary Materials: The following are available online at http:/ /www.mdpi.com/1422-0067/20/5/1171/ s1.

Author Contributions: Conceptualization, Y.-C.W., H.-Y.G. and C.W.; Data curation, Y.-M.W. and H.-Y.G.; Formal analysis, Y.-M.W., C.W. and H.-Y.G.; Funding acquisition, Y.-C.W. and H.-Y.G.; Methodology, Y.-M.W., C.W. and H.-Y.G.; Project administration, Y.-C.W. and H.-Y.G.; Resources, Y.-C.W.; Software, Y.-M.W., C.W. and H.-Y.G.; Writing-original draft, Y.-M.W. and H.-Y.G.; Writing-review and editing, Y.-M.W., Y.-C.W. and H.-Y.G.

Funding: This research was supported by the National Natural Science Foundation of China (31270703 and 31700587), and the Overseas Expertise Introduction Project for Discipline Innovation (B16010).

Acknowledgments: We thank Dong-Lin Guo of Harbin Normal University for the suggestion on the manuscript revision.

Conflicts of Interest: The authors declare that they have no conflict of interest.

\section{References}

1. Gonzalez, A.; Zhao, M.; Leavitt, J.M.; Lloyd, A.M. Regulation of the anthocyanin biosynthetic pathway by the TTG1/bHLH/Myb transcriptional complex in Arabidopsis seedlings. Plant J. 2008, 53, 814-827. [CrossRef] [PubMed]

2. Kang, Y.H.; Kirik, V.; Hulskamp, M.; Nam, K.H.; Hagely, K.; Lee, M.M.; Schiefelbein, J. The MYB23 gene provides a positive feedback loop for cell fate specification in the Arabidopsis root epidermis. Plant Cell 2009, 21, 1080-1094. [CrossRef] [PubMed]

3. Kwon, Y.; Kim, J.H.; Nguyen, H.N.; Jikumaru, Y.; Kamiya, Y.; Hong, S.W.; Lee, H. A novel Arabidopsis MYB-like transcription factor, MYBH, regulates hypocotyl elongation by enhancing auxin accumulation. J. Exp. Bot. 2013, 64, 3911-3922. [CrossRef] [PubMed]

4. Kim, W.C.; Kim, J.Y.; Ko, J.H.; Kang, H.; Han, K.H. Identification of direct targets of transcription factor MYB46 provides insights into the transcriptional regulation of secondary wall biosynthesis. Plant Mol. Biol. 2014, 85, 589-599. [CrossRef] [PubMed] 
5. Kim, W.C.; Ko, J.H.; Han, K.H. Identification of a cis-acting regulatory motif recognized by MYB46, a master transcriptional regulator of secondary wall biosynthesis. Plant Mol. Biol. 2012, 78, 489-501. [CrossRef] [PubMed]

6. Kim, W.C.; Ko, J.H.; Kim, J.Y.; Kim, J.M.; Bae, H.J.; Han, K.H. MYB46 directly regulates the gene expression of secondary wall-associated cellulose synthases in Arabidopsis. Plant J. 2013, 73, 26-36. [CrossRef] [PubMed]

7. Ko, J.H.; Kim, W.C.; Han, K.H. Ectopic expression of MYB46 identifies transcriptional regulatory genes involved in secondary wall biosynthesis in Arabidopsis. Plant J. 2009, 60, 649-665. [CrossRef] [PubMed]

8. Ko, J.H.; Kim, W.C.; Kim, J.Y.; Ahn, S.J.; Han, K.H. MYB46-mediated transcriptional regulation of secondary wall biosynthesis. Mol. Plant 2012, 5, 961-963. [CrossRef] [PubMed]

9. Zhong, R.; Richardson, E.A.; Ye, Z.H. The MYB46 transcription factor is a direct target of SND1 and regulates secondary wall biosynthesis in Arabidopsis. Plant Cell 2007, 19, 2776-2792. [CrossRef] [PubMed]

10. Aoyagi, L.N.; Lopes-Caitar, V.S.; de Carvalho, M.C.; Darben, L.M.; Polizel-Podanosqui, A.; Kuwahara, M.K.; Nepomuceno, A.L.; Abdelnoor, R.V.; Marcelino-Guimaraes, F.C. Genomic and transcriptomic characterization of the transcription factor family R2R3-MYB in soybean and its involvement in the resistance responses to Phakopsora pachyrhizi. Plant Sci. 2014, 229, 32-42. [CrossRef] [PubMed]

11. Su, L.T.; Li, J.W.; Liu, D.Q.; Zhai, Y.; Zhang, H.J.; Li, X.W.; Zhang, Q.L.; Wang, Y.; Wang, Q.Y. A novel MYB transcription factor, GmMYBJ1, from soybean confers drought and cold tolerance in Arabidopsis thaliana. Gene 2014, 538, 46-55. [CrossRef] [PubMed]

12. Pawson, T.; Nash, P. Assembly of cell regulatory systems through protein interaction domains. Science 2003, 300, 445-452. [CrossRef] [PubMed]

13. Fung, S.M.; Ramsay, G.; Katzen, A.L. MYB and CBP: Physiological relevance of a biochemical interaction. Mech. Dev. 2003, 120, 711-720. [CrossRef]

14. Kim, T.; Jung, H.; Min, S.; Kim, K.T.; Ha, H. B-myb proto-oncogene products interact in vivo with each other via the carboxy-terminal conserved region. Febs Lett. 1999, 460, 363-368. [CrossRef]

15. Zimmermann, I.M.; Heim, M.A.; Weisshaar, B.; Uhrig, J.F. Comprehensive identification of Arabidopsis thaliana MYB transcription factors interacting with R/B-like BHLH proteins. Plant J. 2004, 40, 22-34. [CrossRef] [PubMed]

16. Li, D.; Li, Y.; Zhang, L.; Wang, X.; Zhao, Z.; Tao, Z.; Wang, J.; Wang, J.; Lin, M.; Li, X.; et al. Arabidopsis ABA receptor RCAR1/PYL9 interacts with an R2R3-type MYB transcription factor, AtMYB44. Int. J. Mol. Sci. 2014, 15, 8473-8490. [CrossRef] [PubMed]

17. Bhargava, A.; Ahad, A.; Wang, S.; Mansfield, S.D.; Haughn, G.W.; Douglas, C.J.; Ellis, B.E. The interacting MYB75 and KNAT7 transcription factors modulate secondary cell wall deposition both in stems and seed coat in Arabidopsis. Planta 2013, 237, 1199-1211. [CrossRef] [PubMed]

18. Liu, C.; Jun, J.H.; Dixon, R.A. MYB5 and MYB14 Play Pivotal Roles in Seed Coat Polymer Biosynthesis in Medicago truncatula. Plant Physiol. 2014, 165, 1424-1439. [CrossRef] [PubMed]

19. Hu, H.; He, X.; Tu, L.; Zhu, L.; Zhu, S.; Ge, Z.; Zhang, X. GhJAZ2 negatively regulates cotton fiber initiation by interacting with the R2R3-MYB transcription factor GhMYB25-like. Plant J. 2016, 88, 921-935. [CrossRef] [PubMed]

20. Guo, H.; Wang, Y.; Wang, L.; Hu, P.; Wang, Y.; Jia, Y.; Zhang, C.; Zhang, Y.; Zhang, Y.; Wang, C.; et al. Expression of the MYB transcription factor gene BplMYB46 affects abiotic stress tolerance and secondary cell wall deposition in Betula platyphylla. Plant Biotechnol. J. 2017, 15, 107-121. [CrossRef] [PubMed]

21. Chen, Y.; Chen, Z.; Kang, J.; Kang, D.; Gu, H.; Qin, G. AtMYB14 Regulates Cold Tolerance in Arabidopsis. Plant Mol. Biol. Report. 2013, 31, 87-97. [CrossRef] [PubMed]

22. McCarthy, R.L.; Zhong, R.; Ye, Z.H. MYB83 is a direct target of SND1 and acts redundantly with MYB46 in the regulation of secondary cell wall biosynthesis in Arabidopsis. Plant Cell Physiol. 2009, 50, 1950-1964. [CrossRef] [PubMed]

23. Onkokesung, N.; Reichelt, M.; van Doorn, A.; Schuurink, R.C.; van Loon, J.J.; Dicke, M. Modulation of flavonoid metabolites in Arabidopsis thaliana through overexpression of the MYB75 transcription factor: Role of kaempferol-3,7-dirhamnoside in resistance to the specialist insect herbivore Pieris Brassicae. J. Exp. Bot. 2014, 65, 2203-2217. [CrossRef] [PubMed]

24. Park, M.Y.; Kang, J.Y.; Kim, S.Y. Overexpression of AtMYB52 confers ABA hypersensitivity and drought tolerance. Mol. Cells 2011, 31, 447-454. [CrossRef] [PubMed] 
25. Preston, J.; Wheeler, J.; Heazlewood, J.; Li, S.F.; Parish, R.W. AtMYB32 is required for normal pollen development in Arabidopsis thaliana. Plant J. Cell Mol. Biol. 2004, 40, 979-995. [CrossRef] [PubMed]

26. Nie, X.; Ji, X.; Liu, Y.; Zheng, L.; Wang, Y. Elucidation of the specific formation of homo- and heterodimeric forms of ThbZIP1 and its role in stress. Int. J. Mol. Sci. 2014, 15, 10005-10017. [CrossRef] [PubMed]

27. Wang, L.; Zheng, L.; Zhang, C.; Wang, Y.; Lu, M.; Gao, C. ThWRKY4 from Tamarix hispida Can Form Homodimers and Heterodimers and Is Involved in Abiotic Stress Responses. Int. J. Mol. Sci. 2015, 16, 27097-27106. [CrossRef] [PubMed]

28. Zhong, R.Q.; Ye, Z.H. MYB46 and MYB83 Bind to the SMRE Sites and Directly Activate a Suite of Transcription Factors and Secondary Wall Biosynthetic Genes. Plant Cell Physiol. 2012, 53, 368-380. [CrossRef] [PubMed]

29. Zhang, X.; Wang, L.; Meng, H.; Wen, H.; Fan, Y.; Zhao, J. Maize ABP9 enhances tolerance to multiple stresses in transgenic Arabidopsis by modulating ABA signaling and cellular levels of reactive oxygen species. Plant Mol. Biol. 2011, 75, 365-378. [CrossRef] [PubMed]

30. Wang, L.; Qin, L.; Liu, W.; Zhang, D.; Wang, Y. A novel ethylene-responsive factor from Tamarix hispida, ThERF1, is a GCC-box- and DRE-motif binding protein that negatively modulates abiotic stress tolerance in Arabidopsis. Physiol. Plant. 2014, 152, 84-97. [CrossRef] [PubMed]

31. Liu, Y.; Ji, X.; Nie, X.; Qu, M.; Zheng, L.; Tan, Z.; Zhao, H.; Huo, L.; Liu, S.; Zhang, B.; et al. Arabidopsis AtbHLH112 regulates the expression of genes involved in abiotic stress tolerance by binding to their E-box and GCG-box motifs. New Phytol. 2015, 207, 692-709. [CrossRef] [PubMed]

32. Wang, L.; Xu, C.; Wang, C.; Wang, Y. Characterization of a eukaryotic translation initiation factor 5A homolog from Tamarix androssowii involved in plant abiotic stress tolerance. BMC Plant Biol. 2012, 12, 118. [CrossRef] [PubMed]

33. Gampala, S.S.; Hagenbeek, D.; Rock, C.D. Functional interactions of lanthanum and phospholipase D with the abscisic acid signaling effectors VP1 and ABI1-1 in rice protoplasts. J. Biol. Chem. 2001, 276, 9855-9860. [CrossRef] [PubMed]

34. Ji, X.; Zheng, L.; Liu, Y.; Nie, X.; Liu, S.; Wang, Y. A transient transformation system for the functional characterization of genes involved in stress response. Plant Mol. Biol. Report. 2014, 32, 732-739. [CrossRef]

35. Livak, K.J.; Schmittgen, T.D. Analysis of relative gene expression data using real-time quantitative PCR and the 2(-Delta Delta C(T)) Method. Methods 2001, 25, 402-408. [CrossRef] [PubMed]

36. Kim, M.; Ahn, J.W.; Jin, U.H.; Choi, D.; Paek, K.H.; Pai, H.S. Activation of the programmed cell death pathway by inhibition of proteasome function in plants. J. Biol. Chem. 2003, 278, 19406-19415. [CrossRef] [PubMed]

(C) 2019 by the authors. Licensee MDPI, Basel, Switzerland. This article is an open access article distributed under the terms and conditions of the Creative Commons Attribution (CC BY) license (http:/ / creativecommons.org/licenses/by/4.0/). 